\section{International Scientific Journal Theoretical \& Applied Science}

\author{
p-ISSN: 2308-4944 (print) e-ISSN: 2409-0085 (online) \\ Year: $2015 \quad$ Issue: $06 \quad$ Volume: 26
}

Published: $30.06 .2015 \quad$ http://T-Science.org
Zhumabek Shabdenamovich Zhantayev corresponding member of NAS RK, doctor of physical and mathematical Sciences, Professor, JSC "National center of space research and technology" NCA, Republic of Kazakhstan

Nikolay Gergiyevich Breusov candidate of physico-mathematical Sciences, JSC "National center of space research and technology"

NCA, Republic of Kazakhstan

\section{Galina Yakovlevna Khachikyan} prof., DrSc,

SLLP "Institute of ionosphere" JSC NCCIT NCA,

Republic of Kazakhstan galina.khachikyan@gmail.com

Kanat Mukashevich Mukashev prof., DrSc,

Al-Farabi Kazakh National University mukash_kanat@mail.ru

Turlan Khamzinovich Sadykov prof., DrSc,

Physico-Technical Institute

JSC "NNTH Parasat" MES, Republic of Kazakhstan

\title{
SOLAR ACTIVITY AND SEISMICITY OF THE EARTH IN THE NORTHERN TIEN - SHAN
}

\begin{abstract}
Work is devoted to the discussion of the experimentally observed conformity between the intensity of cosmic rays and variations in solar activity and characteristics of seismicity in the Northern Tien Shan. Explanation of this fact is based on the analysis of the results of theoretical studies on the impact on the terrestrial rocks of the penetrating component of cosmic rays -the muons. Under such exposure, microcracks can be formed in the rocks which is accompanied by the appearance of acoustic waves. If this reaction of muons occurs with highly stressed seismically active medium, the energy seismic waves emanates can increase by more than 20 orders of magnitude.This may cause not only the generation of an acoustic wave but also, of a seismic wave associated with an earthquake.

Key words: extensive air showers, muons, three dimensional stress, seismically active environment.

Language: Russian

Citation: Zhantayev ZS, Breusov NG, Khachikyan GY, Mukashev KM, Sadykov TK (2015) SOLAR ACTIVITY AND SEISMICITY OF THE EARTH IN THE NORTHERN TIEN - SHAN. ISJ Theoretical \& Applied Science 06 (26): 5-11.

Soi: http://s-o-i.org/1.1/TAS*06(26)2 Doi: crossef http://dx.doi.org/10.15863/TAS.2015.06.26.2

\section{СОЛНЕЧНАЯ АКТИВНОСТЬ И СЕЙСМИЧНОСТЬ ЗЕМЛИ В УСЛОВИЯХ СЕВЕРНОГО ТЯНЬ- ШАНЯ}

Аннотация: Работа посвящена обсуждению экспериментально наблюдаемого соответствия между интенсивностью потока космического излучения и вариациями солнечной активности и характеристик сейсмичности на территории Северного Тянь-Шаня. Объяснение данного факта осуществляется на основе анализа результатов исследований, посвященных воздействию на земные породы проникающей компоненты космических лучей - мюонов. При таком воздействии в породах могут образовываться микротрещины, возникновение которых сопровождается генерацией акустических волн. Если данное взаимодействие мюонов происходит с высоконапряженной сейсмически активной средой, излучаемая сейсмическими волнами энергия может возрасти на десятки порядков. Это может привести к генерации не только акустической, но и сейсмической волны, связанной с землетрясением.
\end{abstract}

ISPC Education \& Innovation, 
Ключевые слова: широкие атмосферные ливни, мюоны, объемное напряженное состояние, сейсмоактивная среда.

Введение. Под космическими лучами (КЛ) обыкновенно понимаются потоки заряженных релятивистских частиц, начиная от протонов и ядер гелия и кончая ядрами более тяжёлых элементов вплоть до урана, рождённых и ускоренных до высоких и предельно высоких (вплоть до $10^{20}$ эВ) энергий вне пределов Земли. При этом в потоке частиц с энергией до $10^{9}$ эВ доминирует вклад Солнца, а частицы более высоких энергий имеют галактическое (и, возможно, при самых высоких энергиях экстрагалактическое) происхождение. Естественно, что протоны и ядра не исчерпывают всего многообразия излучений, приходящих на Землю из космического пространства.

В составе галактических космических лучей (ГКЛ) преобладают протоны, на долю остальных ядер приходится менее 10\%. Протоны остаются доминирующей компонентой, по крайней мере, до энергий $\sim 1$ ТэВ, хотя доля ядер возрастает с увеличением энергии частиц. В составе ГКЛ содержание лёгких ядер (с зарядом Z от 3 до 5) на несколько порядков превосходит их содержание в звёздах. Кроме того, ГКЛ характеризуются значительно большим присутствием тяжёлых ядер $(\mathrm{Z}>20)$ по сравнению с их естественной распространённостью. Аномально высокая представленность этих элементов связана с дополнительным вкладом от расщепления более тяжёлых элементов в межзвёздной среде. Оба эти фактора весьма существенны для выяснения вопроса о происхождении ГКЛ.

Солнце также является источником КЛ, и потоки солнечных космических лучей (СКЛ), особенно во время солнечных вспышек, могут достигать очень больших значений, однако характерная величина их энергии, как правило, не превосходит $10^{9} э \mathrm{~B}$, тогда как ГКЛ распределены в очень широком диапазоне энергий от $10^{9}$ до $10^{20}$ эВ. Поэтому разделение КЛ на галактические и солнечные отражает существо дела, поскольку как характеристики, так и источники СКЛ и ГКЛ, совершенно различны. При энергиях ниже 10 ГэВ/нуклон интенсивность ГКЛ, измеряемая вблизи Земли, зависит от уровня солнечной активности (точнее, от меняющегося в течение солнечных циклов магнитного поля). В области более высоких энергий интенсивность ГКЛ постоянна во времени. Согласно существующим представлениям, собственно ГКЛ заканчиваются в области энергий между $10^{17}$ и $10^{18}$ эВ. Поэтому при энергиях выше $10^{18}$ эВ более правильно использовать обозначение просто КЛ, поскольку происхождение космических лучей предельно высоких энергий, скорее всего, с Галактикой не связано. Экспериментально наблюдаемый дифференциальный энергетический спектр КЛ (Cronin, 1999) показан на рис.1.

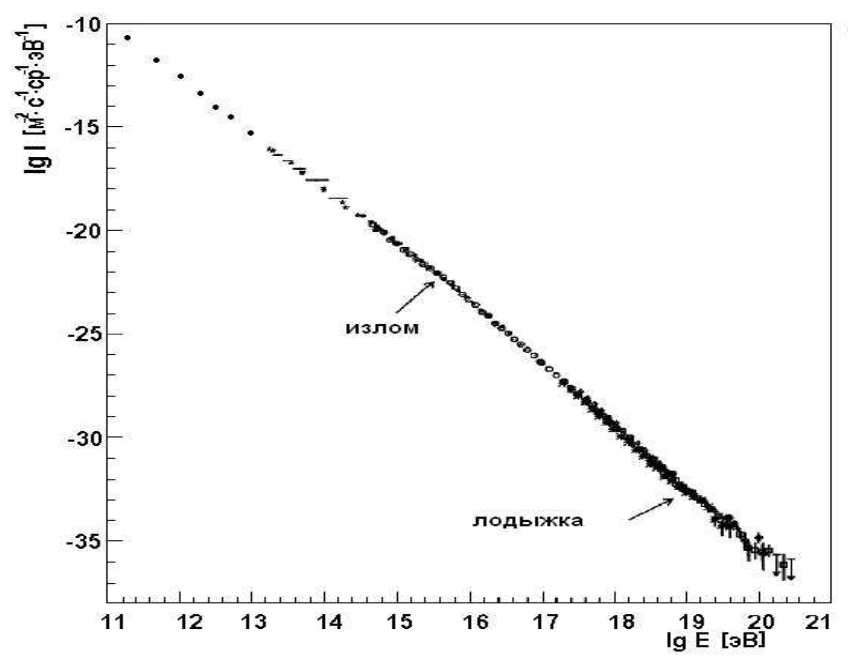

Рисунок 1 - Энергетический спектр космических лучей.

Спектр описывается степенным законом в очень широком диапазоне энергий от $10^{11}$ до $10^{20}$ эВ с небольшим изменением наклона около $3 \cdot 10^{15}$ эВ (излом, иногда называемый коленом, knee) и около $10^{19}$ эВ (ankle, лодыжка). Интегральный поток КЛ выше лодыжки равен приблизительно 1 частице на км ${ }^{2}$ в год.
Степенной характер энергетического спектра КЛ свидетельствует о нетепловом происхождении их энергии, а это, в свою очередь, налагает определённые требования на источники КЛ, которые должны обеспечить формирование степенного энергетического спектра. Максимальная энергия частиц КЛ, которая была

ISPC Education \& Innovation, 
зарегистрирована по наблюдениям широких атмосферных ливней, составляет $3.10^{20}$ эВ и были зарегистрированы более 10 событий, энергия которых $>10^{20} э \mathrm{~B}$. Полагают, что такие энергии вряд ли могут быть обеспечены источниками, находящимися в нашей Галактике.

Сущность проблемы. Как известно, на Земле ежегодно происходит примерно 18-20 землетрясений с магнитудой 7,0 и выше по шкале Рихтера. В результате этих явлений погибают более 60000 человек. В этих условиях экономический ущерб достигает сотни миллиардов долларов США. Поэтому естественно возникает высокий социальный спрос на надежные методы прогноза землетрясений. Территория Казахстана в этом плане не является исключением, поскольку около третьи его площади расположено в сейсмоопасных районах. В свое время в этих районах происходили разрушительные землетрясения и, очевидно, они не исключены в будущем [1]. С механической точки зрения, землетрясение представляет собой мгновенную разрядку накопившегося в конкретном объеме геологической среды напряжения, поэтому одним из ключевых факторов для сейсмического прогноза является информация об объемном напряженном состоянии (ОНС) геологической среды в районе потенциально возможного очага землетрясения, расположенного, как правило, на глубине порядка 10 км и более. Ранее была высказана идея о возможности мониторинга ОНС среды на глубине формирования очагов землетрясений с использованием мюонного потока космических лучей, проникающего на несколько километров вглубь земной коры [2]. Сущность идеи заключается в том, что при воздействии высокоэнергичных мюонов на сейсмически активную среду, напряжение в которой близко к критическому (порогу разрушения), может произойти мгновенный сброс критического напряжения, то есть, поток космических лучей может сыграть роль спускововго механизма землетрясения. Поэтому актуальность систематизации статистических результатов о соответствии между вариациями космических факторов и характеристик сейсмичности, как на всей планете, так и на территории Северного Тянь-Шаня, несомненна.

Важнейшие характеристики космических лучей. Приходящие к Земле первичные космические лучи с энергией частиц $\mathrm{E}_{\mathrm{o}}>10^{13}$ эВ порождают в атмосфере каскад вторичных частиц, так называемый широкий атмосферный ливень (ШАЛ). Расчеты каскада вторичных частиц, основанные на методе Монте-Карло по программе CORSIKA (COsmic Ray SImulations for KAscade) показали, что максимум ШАЛ приходится на высоту порядка 15 км. На практике, структуру ШАЛ можно представить в виде тонкого диска, состоящего из отдельных частиц (пионов, электронов, протонов, нейтронов, мюонов и нейтрино) движущихся со скоростью, близкой к скорости света, по направлению первичной частицы. На этой высоте доминирующей является электроннофотонная компонента. Примерно $10 \%$ от числа всех электронов составляют мюоны, количество которых зависит от энергии первичной частицы. Компьютерное моделирование показало, что при энергии $\mathrm{E}_{0}=10^{16}$ эВ, в ШАЛ может содержаться примерно 4 мюона с энергией $\mathrm{E} \geq 3$ ТэВ, а при энергии $\mathrm{E}_{0}=10^{19}$ эВ их число с той же энергией достигает более 1500. Проходя через атмосферу и приближаясь к земной поверхности, частицы ШАЛ теряют свою энергию в основном в процессе ионизации, а также тормозного излучения, образования пар и фотоядерных взаимодействий, называемые радиационными процессами. Поскольку масса мюона почти в 200 раз больше массы электрона, эффективность взаимодействия мюонов с атмосферными газами в $4.10^{4}$ раз меньше, чем у электронов. В результате на уровне земной поверхности поток мюонов становится доминирующим. Согласно современным данным на значительную глубину в земную кору проникают только мюоны и нейтрино. Мюоны теряют энергию в результате взаимодействия в ионизационных и радиационных процессах. Полная потеря энергии мюона может быть выражена в виде функции от массы пройденного вещества как:

$$
-d E_{\mu} / d X=a+b E_{\mu},
$$

где $a$ - ионизационные потери, $b$ - потери за счет радиационных процессов. Оба эти параметра в определенной степени зависят от энергии частиц. Значение $a / b \approx 500 \mathrm{GeV}$ в стандартной горной породе определяет критическую энергию, ниже которой ионизационные потери становятся преобладающими над радиационными. На рисунке 2 показаны результаты расчета и измерения интенсивности мюонов в земной коре до глубин более 100 км водного эквивалента (1 км в.э. $=10^{5}$ г.см-2 стандартной горной породы) при условии пренебрежения энергетической зависимостью отношения $a / 8$ [3].

Линиями показаны результаты расчетов с использованием программы CORSIKA, a символами - данные измерений в разных экспериментах $[4,5]$. Серая область на больших глубинах представляет мюоны с энергией более 2 ГэВ, которые были индуцированы при взаимодействии с нейтрино. Верхняя линия - для горизонтальных потоков индуцированных мюонов, нижняя - для мюонов, распространяющихся вертикально вверх. Более 
темно закрашенная область показывает мюонный поток, измеренный в эксперименте SuperKamiokande, который расположен в горах Японии на глубине 1 км под землей и имеет детектор в виде огромного резервуара (40м х 40м) из нержавеющей стали, который заполнен $5.10^{4}$ тонн чистой воды, служащей в качестве мишени для нейтрино.

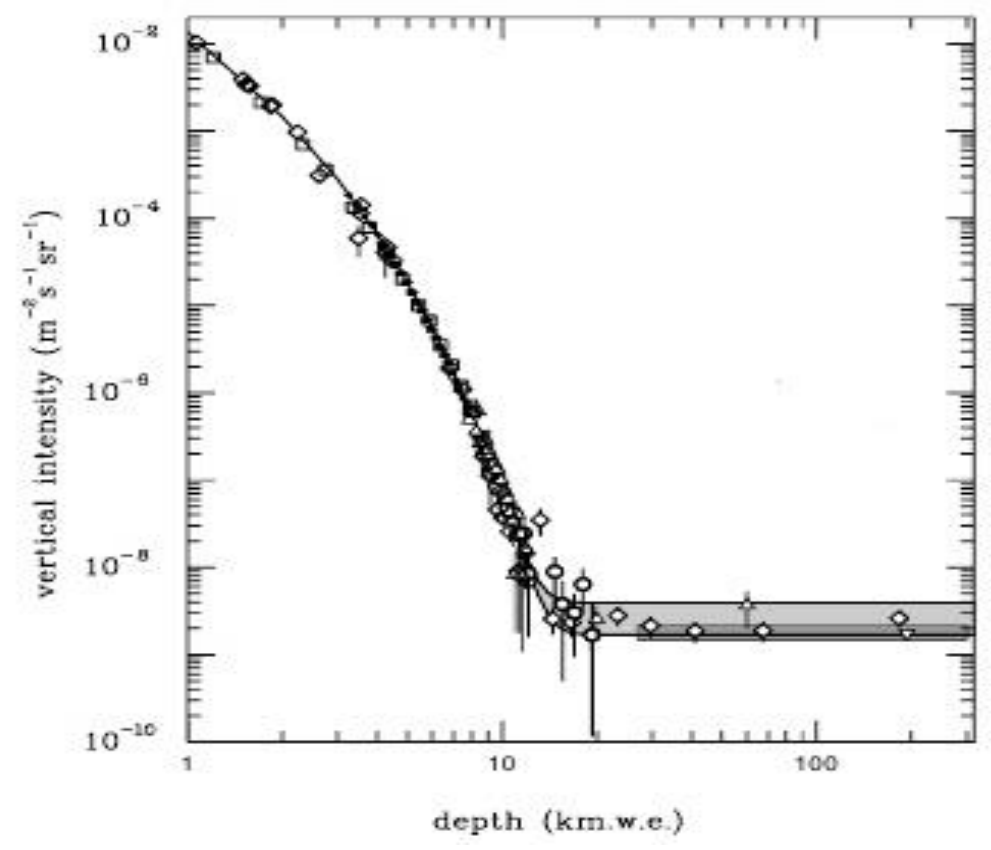

Рисунок 2 - Интенсивность потока мюонов в зависимости от глубины в единицах водного эквивалента [3].

Солнечная активность и сейсмичность на Северном Тянь-Шане. Именно указанные выше результаты явились основанием для постановки вопроса о возможности влияния мюонов на процессы в земной коре. Так, в работе [6] были проанализированы 11 извержений за последние 306 лет $(1700$ - 2005гг.) четырех вулканов на территории Японии (Fuji, Usu, Mуojinsho, Satsuma-Iwo-jima). Обнаружено, что 9 из 11 извержений произошли на фазе минимума солнечной активности, когда интенсивность потока космических лучей, в том числе мюонов, ожидается максимальной. Однако, такой связи не было обнаружено для извержений другого японского вулкана - Izu-Ohshima. Анализ состава магмы в этих вулканах показал наличие существенных различий между ними. Следовательно, не для всех вулканов Японии время их извержения тяготеет к минимумам солнечной активности, когда присутствует повышенный поток космических лучей. Это говорит о том, что земные породы могут избирательно реагировать на воздействие солнечной активности и/или потока космических лучей - в зависимости от их геологического строения.

В поддержку такой избирательной реакции свидетельствуют и результаты почти десятилетнего изучения связи сейсмотектонической деформации земной коры на Северном Тянь-Шане с вариациями солнечной активности, обзор результатов которых приведен в работе [7]. По данным о механизмах очагов землетрясений с энергетическим классом $\mathrm{K}=7-9$, за период 1982-2012гг. установлено, что только на локальной территории, расположенной в высокогорной части хребтов Заилийский и Кунгей Алатау, между Алматинской впадиной на севере и Иссыкульской - на юге, Аксайским и Тургеньским разломами - на западе и востоке, режим сейсмотектонической деформации земной коры коррелирует с вариациями солнечной активности. Она находится в состоянии одноосного сжатия в годы низкой солнечной активности, но одноосного растяжения в годы высокой солнечной активности. Как показано в [8], строение литосферы этого локального района отличается от соседних. А именно, результаты скоростного моделирования литосферы по субширотному Тянь-Шаньскому геотраверсу показывают, что земная кора данного локального района подстилается наиболее мощным слоем активной мантии (горячим мантийным потоком) и, как показывает магнитотеллурическое зондиро-вание, низкоомные (высокопроводящие) объемы земных пород территориально совпадают с горячим мантийным потоком [9].

Данные, подтверждающие эти результаты и свидельствующие о связи сейсмичности на Северном Тянь-Шане с вариациями солнечной 
активности, получены также в ряде других работ $[10,11]$. На территории Северного Тянь-Шаня за последние 130 лет произошло восемь сильных землетрясений [12]: Беловодское (1885г., $M=6,9)$; Верненское (1887г., M=7,3); Чиликское (1889г., $M=8,3)$; Кеминское (1911г., $M=8.2)$; КеминоЧуйское (1938г., $M=6,9)$; Сарыкамышское (1970г., $M=6,8)$; Жаланаш-Тюпское $\quad(1978$ г., $\quad M=6,8)$; Байсорунское $(1990 г$., $M=6,4)$. Анализ этих событий в связи с вариациями солнечной активности выявил [13] четкую обратную зависимость между их магнитудой и среднегодовым числом солнечных пятен $(W)$ (рисунок 3). Сплошная прямая на рисунке есть линейная аппроксимация наблюденных данных с уравнением регрессии:

$M=7,96-0,012 W$, среднеквадратическим отклонением $\mathrm{SD}=0,36$ и коэффициентом корреляции $\mathrm{R}=-0,88$. Поразительно, но как и для случая мощных землетрясений в Японии, сильнейшие землетрясения на Северном ТяньШане также произошли в годы минимумов солнечной активности.

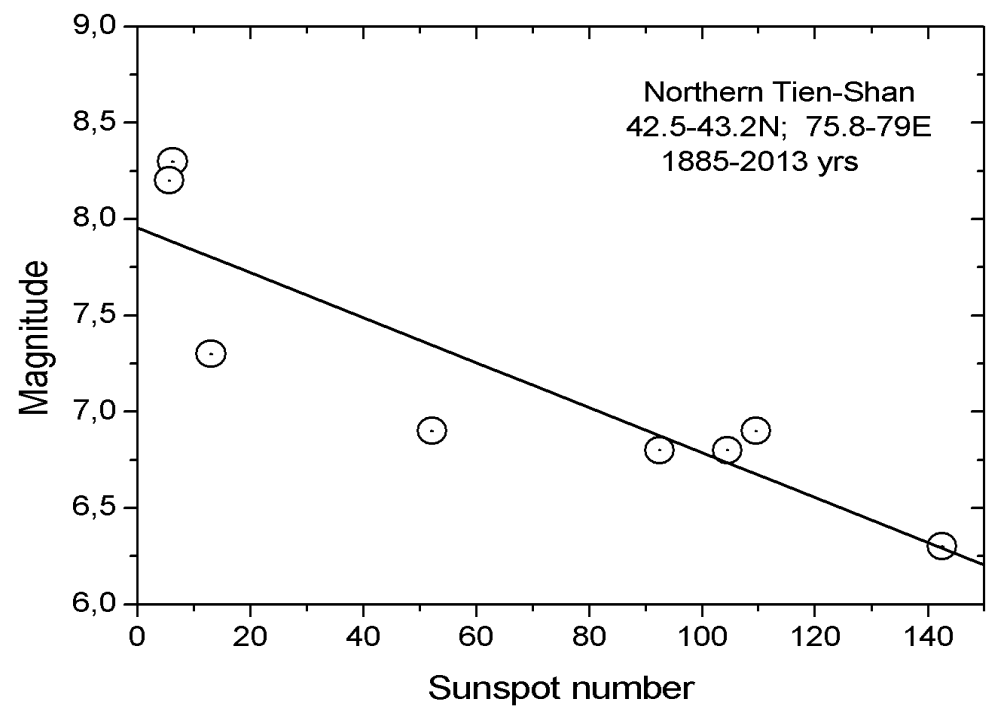

Рисунок 3 - Магнитуды сильных землетрясений, произошедших на Северном Тянь-Шане за последние 130 лет, в зависимости от числа солнечных пятен [13].

Этот факт демонстрирует рисунок 4, где приведены среднегодовые числа солнечных пятен за период с 1750 по 2014гг (1-24 солнечные циклы), а красными звездочками отмечены даты четырех сильнейших землетрясений на Северном Тянь-Шане. За период инструментальных наблюдений за Солнцем идентифицировано 24 солнечных 11-ти летних циклов (номера в нижней части рисунка 4). Видно, что амплитуды 11-ти летних циклов не постоянны, что приводит к появлению долговременных солнечных максимумов и минимумов. В литературе активно обсуждается минимум Маундера (16451715гг), установленный по палеоданным, а за инструментальный период наблюдений зафиксировано еще два солнечных минимума $[14,15]$ : Дальтона, середина которого пришлась примерно на 1813г. (5-7 солнечные циклы), и Гляйсберга, середина которого пришлась примерно на 1910 г., а самыми низкоамплитудными были 12-ый и 14-ый циклы. Из рисунка 5 видно, что четыре землетрясения, разрушивших г. Алматы, произошли в периоды долговременных солнечных минимумов Дальтона и Гляйсберга, при этом, они были приурочены к годам солнечных минимумов, завершавших низкоамплитудные 11-ти летние циклы (5-ый, 12-ый и 14-ый). 


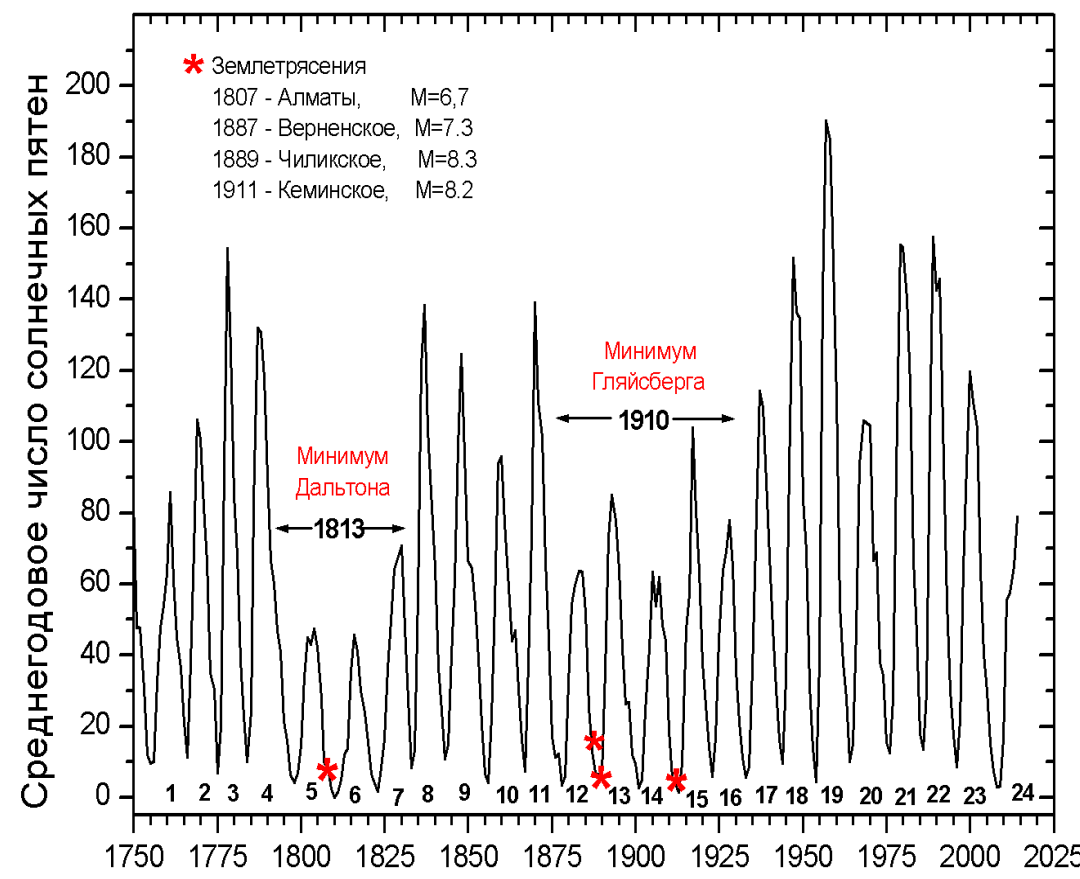

Годы

\section{Рисунок 4 - Среднегодовые числа солнечных пятен в 1750-2014 гг (1-24 циклы) и даты сильнейших землетрясений на Северном Тянь-Шане (звездочки).}

Также, для данной территории характерно наличие в подкоровом пространстве активной мантии. Она характеризируется низкими значениями скорости $\left(V_{p}=7,6\right.$ км/с) и аномально высокой температурой. На глубине 280-160км тепломассопотоки локализованы в так называемые плюмовые каналы, а по мере прослеживания вверх их горизонтальное сечение увеличивается и, фактически, земная кора этой территории расположена непосредственно над плюмовым каналом - в зоне подкорового латерального «растекания» мантийного субстрата, где его температура достигает 1000$1300^{\circ} \mathrm{C}[16]$. С учетом отмеченных тектонических особенностей строения литосферы данного района, можно утверждать о том, что на локальных территориях, где имеются подстилающие мантийные плюмы, возможно относительное растяжение (уменьшение сжатия), фиксируемое многочисленными землетрясениями со сбросовыми механизмами в очагах, в отличие от большинства вбросовых механизмов в окружающем пространстве.

Вероятно, этим самым можно объяснить соответствие между вариациями сейсмотектонической деформации и солнечной активности. Но это возможно при условии, что солнечная активность влияет на активность подстилающей земную кору мантии или, другими словами, на активность мантийных плюмовых каналов. Открытым остается вопрос о физическом механизме возможного влияния солнечной активности на мантийный субстрат. Не исключено, что физический механизм такого влияния может быть лишь составной частью более общего механизма солнечно-земных связей, который пока еще до конца не разработан, и в котором одним из ключевых параметров являются именно космические лучи.

T.o., анализ приведенных статистических результатов о соответствии между вариациями солнечной активности, интенсивности потока космического излучения и вариациями характеристик сейсмичности Земли с позиции современного взгляда на физический механизм солнечно-земных связей показывает, что одним из ключевых параметров в данном случае могут выступить космические лучи.

Представленные в данной статье результаты обсуждались на заседаниях соответствующих секций двух международных симпозиумов $[17,18]$. 


\section{References:}

1. Sydykov A (2004) Seysmicheskiy rezhim territorii Kazakhstana. -Almaty: Gylym. 2004. $270 \mathrm{p}$.

2. Tsarev VA, Chechin VA (1988) Atmosfernye myuony i vysokochastotnye seysmicheskie shumy. Preprint FIAN. - 1988.- № 179. - 21p.

3. Beringer J, et al. (2013) Particle Data Group, PR D86, 010001 (2012) and 2013 partial update for the 2014 edition. -2013, - December 18 . Available:: http://pdg.lbl.gov (Accessed: 21.05.2015).

4. Adamson P, et al. (2007) Measurement of the atmospheric muon charge ratio at $\mathrm{TeV}$ energies with MINOS //Phys. Rev.-2007. - Vol. D76. pp. 052003. - hepex/0705.3815.

5. Ambrosio M, et al. (1995) (MACRO Collab.). Vertical muon intensity measured with macro at the Ggran Sasso laboratory. // Phys. Rev. 1995. - D52. - pp. 3793-3802.

6. Ebisuzaki T, Miyahara H, Katoaka R, Sato T, Ishimine Y (2011) Explosive volcanic eruptions triggered by cosmic rays: Volcano as a bubble chamber // Gondwana Research. 2011. - V.19. pp. 1054 - 1061.

7. Abakanov TD, Sadykova AB, Khachikyan GY (2015) Sovremennoe seysmotektonicheskoe sostoyanie zemnoy kory na Severnom Tyan'Shane. Doklady Natsional'noy Akademii Nauk Respubliki Kazakhstan. ISSN 2224-5227. -2015. - № 2. - pp. 98-110.

8. Timush AV, Sadykova AB, Stepanenko NP, Khachikyan GY (2013) Stroenie litosfery kak faktor variatsiy seysmotektonicheskikh deformatsiy v svyazi s solnechnoy aktivnost'yu na Severnom Tyan'-Shane //Izvestiya NAN RK, seriya geologii i tekhnicheskikh nauk. -2013, № 1. - pp. 55-66.

9. Rybin AK, Batalev VY, Shchelochkov GG, et al. (2002) Na puti k 3D strukture zemnoy kory i verkhney mantii Tyan'-Shanya: rezul'taty glubinnoy magnitotelluriki //Geodinamika i geoekologicheskie problemy vysokogornykh regionov (2-y mezhdunarodnyy simpozium; tezisy). Bishkek, 2002. pp.25-27.

10. Poleshko NN, Sadykova AB, Sydykov A, Timush AV, Khachikyan GY, Shatsilov VI (2009) Variatsii solnechnoy aktivnosti i seysmotektonicheskie deformatsii na Severnom Tyan'-Shane: Chast' 1. Koeffitsient Lode-Nadai //Vestnik NYaTs RK. - 2009. -Vyp.1. - pp. 8692.
11. Timush AV, Sadykova AB, Stepanenko NP, Khachikyan GY (2013) Stroenie litosfery kak faktor variatsii seysmotektonicheskikh deformatsiy v svyazi s solnechnoy aktivnost'yu na Severnom Tyan'-Shane //Izvestiya NAN RK. Seriya geologii i tekhnicheskikh nauk. 2013. №1. -pp.55-66.

12. Kal'met'eva ZA, Mikolaychuk AV, Moldobekov BD, Meleshko AV, Zhantaev MM, Zubovich AV (2009) Atlas Zemletryaseniy Kyrgyzstana. Bishkek - TsAIIZ - 2009. - ISBN 978-9967-25-829-7. - 74 p.

13. Khachikyan GY, Sadykova AB, Poleshko NN (2014) Variatsii solnechnoy aktivnosti i seysmotektonicheskaya aktivnost' Severnogo Tyan'-Shanya. //Mezhdunarodnyy nauchnyy zhurnal-prilozhenie Respubliki Kazakhstan. Vysshaya shkola Kazakhstana. Poisk-Izdenis.2014. - №2(1) - pp. 114-119.

14. Feynman J, Ruzmaikin A (2011) The Sun's Strange Behavior: Maunder Minimum or Gleissberg Cycle? // Solar Phys . 2011 V.272. P. 351-363. DOI 10.1007/s11207-0119828-0.

15. Feynman J, Ruzmaikin A (2014) The Centennial Gleissberg Cycle and its association with extended minima // J. Geophys. Res. Space Physics. 2014. V.119. pp.6027-6041. doi:10.1002/2013JA019478.

16. Shatsilov VI, Gorbunov PN, Timush AV (2000) Novye dannye o tektonosfere Tyan'Shanya //Doklady NAN RK. - 2000. - №2. pp.50-54.

17. Zhantayev Z, Khachikyan G, Breusov N (2014) On dependence of seismic activity on 11 year variations in solar activity and/or cosmic rays. Geophysical Research Abstracts. 2014. V.16.EGU2014-5253. Presentation. Available: http:/meetingorganizer.copernicus.org/EGU201 4/orals (Accessed: 21.05.2015).

18. Sadykov TK, Zhukov VV, Breusov NG, Mukashev KM, Khachikyan GY, Zastrozhnova NN (2013) Seismic stations for short-term prediction of earthquakes by means of the cosmic rays // Materialy VIII mezhdunarodnoy nauchno-prakticheskoy konferentsii «Novosti nauchnoy mysli - 2013». Izd: Publishing House «Education and Science» (Chekhiya, Praga). 28-30 October 2013. Available: www.rusnauka.com/CONF/NEW_CONF/1.htm (Accessed: 21.05.2015). 